\title{
Smart Placement of Motion Sensors in a Home Environment
}

\author{
Maria Pia Fanti, Michele Roccotelli \\ Department of Electrical and Information \\ Engineering \\ Politecnico di Bari \\ Bari, Italy \\ \{mariapia.fanti, michele.roccotelli\}@poliba.it
}

\author{
Gregory Faraut, Jean-Jacques Lesage \\ LURPA - ENS \\ Cachan, University of Paris-Sud, Université \\ Paris-Saclay, \\ 94235 Cachan, France \\ \{gregory.faraut, jean-jacques.lesage\} @ens-paris- \\ saclay.fr
}

\begin{abstract}
This paper deals with the smart placement of motion sensors in smart homes for Ambient Assisted Living, by considering the sensor technology and cost and respecting specific coverage requirements. The core of the proposed methodology is a decision module that can optimize the sensors placement according to different objectives. More precisely, the main objective is the minimization of costs of the deployed sensors. Moreover, the second objective can be the maximization of the overlapping in order to find a robust solution or the minimization of the overlapping of the detection areas in order to improve the inhabitant localization. A case study demonstrates the effectiveness of the proposed strategy on sensors placement in a domestic environment.
\end{abstract}

Keywords - Smart Sensor Placement, Optimization, Integer Linear Programming, Smart Home.

\section{INTRODUCTION}

In recent years, Ambient Assisted Living (AAL) applications have found large diffusion in smart home environment [1]-[3], [6], [7], [13], [14]. The main goal of AAL is improving the comfort, the health and the safety of aged or disabled people living in their own house. Smart technologies and devices, like sensors, actuators and controllers, in combination with smart strategies are largely used to satisfy the AAL goals. In this context, several AAL applications are included such as the control of smart equipment to support elderly people in their daily activities, the remote monitoring of patients having long-term diseases, and the indoor location tracking of inhabitants [1], [6]-[15]. Among all the applications, the tracking of people movements at home is an important task both for comfort, healthcare and safety purposes [1]-[3], [6]-[9]. To ensure an optimal location tracking performance by properly covering the spaces and minimizing the cost of the instrumentation, it is important to optimize the sensors deployment [1]-[5], [13]. Therefore, the optimization of sensors deployment has been one of the main objective in different kind of environments, like residential, office and public buildings or areas for improving the quality of the provided services. The quality of these services depends also on accurate occupants localization and movement analysis. For instance, a system that implements an accurate location-tracking method can improve the inhabitants living experience as it can anticipate and provide various intelligent services based on the occupants location and movement trajectory [3].

It is remarked that the effectiveness of a methodology for location tracking and for space coverage depends not only by the tracking technology but also by the localization model and the geometric and structural properties of the deployment space [1][3], [6].

In a domestic context, the localization of occupants through the detection of their movements is performed by using different kind of sensors and systems [1]-[4], [8]-[15].

Some authors propose tracking systems based on wearable and cameras devices that are considered invasive sensors [8][11]. Wearable sensor systems allows localizing people positions with good accuracy but there are several drawbacks derived from their use. For instance, aged people may forget to follow the instructions and prefer to not wear or carry invasive devices. In addition, many of these systems require complex infrastructure to be installed at home and there are also privacy issues to be considered [8], [10].

Other contributions refer to location tracking performed by using robotic systems [9], [11], [12]. These kind of systems are also invasive, expansive and their use has been mainly demonstrated by simulations rather than real life experiments.

On the other hand, to localize the inhabitant positions or activities in real time avoiding privacy issues, using cheap and easy-to-install sensors, that do not requires for the human interaction, Passive Infrared (PIR) motion sensors can be used [1], [3], [4], [6], [7], [13]-[15]. They are low-cost, non-intrusive sensors that are activated when a movement is performed within their detection area. Nowadays, the use of wireless PIR sensors is preferred due to their flexibility and easiness of installation [13]-[15].

In this context, considering the relevance of the instrumentation cost when implementing smart home technologies, the necessities to cover the spaces and to locate the people with high accuracy, this paper investigates the possibility of minimizing PIR sensors numbers (and consequently cost), guaranteeing specific coverage requirements related to location tracking purposes. Given a home map and a database of PIR sensors, our goal is to provide a decision-making tool helping the experts to do the best choice for sensors deployment, considering their technologies and costs, the geometric definition of the spaces and the coverage and localization necessities.

\section{A. Paper contribution}

The paper presents a methodology for the smart placement of motion sensors inside a known environement that provides optimal solutions for sensor deployment, minimizing sensors 
cost and satisfying location tracking purposes under zone coverage constraints.

More in detail, the contribution of the paper with respect to the state of the art can be resumed as follows:

i. Compared to [8]-[11], our methodology is based on the use of non-intrusive, low-cost and easy-to-install sensors that do not depend by human collaboration.

ii. With respect to [1] and [2] the authors improve the model of the decision-making system by providing an updated version of the database module that includes new sensors technological aspects. In addition, the optimization phase has also been improved. In this phase of the proposed methodology is now possible to provide a solution for different optimization objectives, such as minimizing the cost of sensors, minimizing or maximizing the overlapping of their detection areas, always respecting coverage constraints. The optimized solutions include also the optimal orientation to be considered when installing sensors.

The rest of the paper is organized as follows. Section II presents the methodology for sensor deployment optimization and Section III describes the optimization phase of the methodology. Moreover, Section IV proposes a case study based on a real home map. Finally, Section V summarizes the conclusions and introduces the perspectives for further works.

\section{SENSOR PlacEMENT PROBLEM}

In this section, the PIR sensor placement problem is presented by describing the detection capacities of such sensors, their placement and use in a generic home area.

As previously cited, PIR motion sensors are able to detect the movements of people. In this paper, two kinds of PIR sensors are studied: wall sensors and ceiling sensors. The first are sensors to be installed on walls and the seconds are sensors to be installed on ceilings. Basically, these sensors are characterized by a detection capacity depending by two parameters [1]: a) detection angle $d a\left[^{\circ}\right]$ and b) a detection radius $d r[m]$. Fig. 1 provides a sketch of the detection area of a ceiling sensor with detection angle $d a=360^{\circ}$ and $d r=R, \quad R \in \mathbb{N}^{+}$. The placement of such a sensor, as well as its coverage area, is strongly influenced by the space geometry. Indeed, the presence of walls, partitions, doors, windows and furnishings can constitute an obstacle for the sensor detection, generating a dark zone that is an uncovered zone within its detection area (Fig. 1). In addition, the detection area of a motion sensor depends also on its orientation. Indeed, if a sensor is not well oriented with respect to the area that must be covered, the coverage objective will not be satisfied.

Therefore, three technological aspects of such sensors are taken into account when dealing with the sensor placement problem: i) the distance of detection of the sensor (detection radius); ii) the angle of detection of the sensor; iii) the orientation of the sensor.
Hence, it is important to determine the proper detection radius, angle and orientation of the sensor to be installed to satisfy the coverage requirements, as it is shown in Fig. 2.

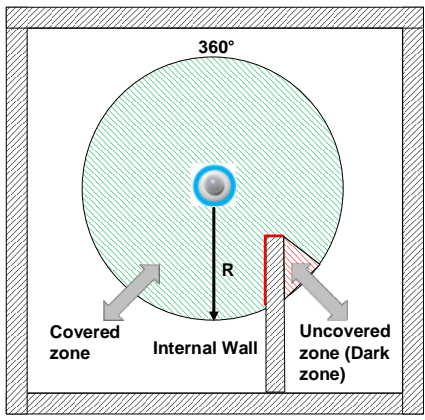

Fig. 1. The detection area of a $360^{\circ}$ ceiling sensor influenced by an obstacle.

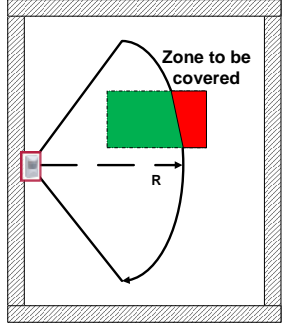

(a)

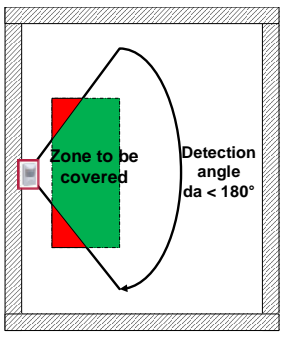

(c)

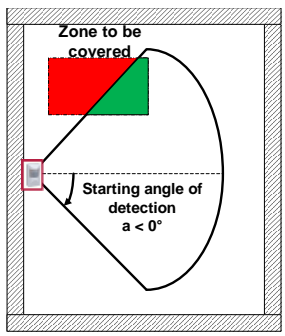

(e)

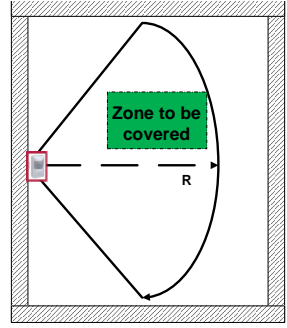

(b)

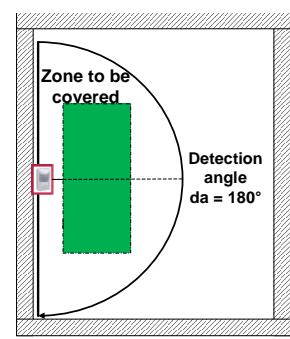

(d)

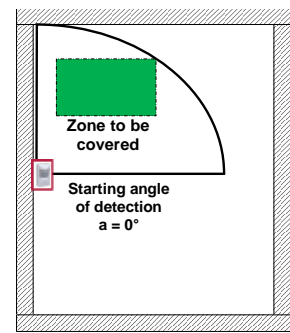

(f)
Fig. 2. The determination of the right detection radius (a)-(b), detection angle (c)-(d) and orientation (e)-(f) of a wall motion sensor when covering a target zone.

Given a home map and a set of sensors, the designer problem is to decide which sensors have to be used, on the basis of their technology, where and how to install them, by minimizing the costs and respecting specific requirements. Therefore, the goal is to provide a tool able to optimize the sensors deployment by considering their technical capacity and cost, the dwelling 
topology and by respecting specific localization and coverage constraints.

\section{SMART Placement Methodology}

In this section, the methodology for the smart placement of motion sensors is presented. The implementation of the proposed methodology is demanded to an updated version of the integrated system for decision-making presented in [1]. In particular, the decision-making system has a modular structure and it is composed by: i) a database module to store and provide technical data about the home maps and the available sensors; ii) a decision (operational) module to provide the system with the optimal sensor placement solutions according to specific requirements.

Fig. 3 depicts the integrated system that implement the sensor placement methodology composed by different phases.

The database module contains a home database and a sensor database. The first database provides the home map with structural information, including positions of walls and of the installed furnishings. The second database provides information on sensors of different types including their detection capacities and market costs.

Let us consider a set of sensor $H=H_{w} \cup H_{c}$, with cardinality $S$, that is given by the union of two sets $H_{w}$ and $H_{c}: H_{w}$ is the set of wall sensor types and $H_{c}$ is the set of ceiling sensor types. Moreover, each element $h \in H$ with $h \in \mathbb{N}^{+}$denotes a sensor type and is characterized by its detection capacity: detection radius $d r_{h}[m]$ and detection angle $d a_{h}\left[^{\circ}\right]$.

The decision module is the core of the decision-making system and it works according to a three operational phases procedure: 1) classification phase; 2) discretization phase; 3) optimization phase.

The classification phase identifies the zones of the dwelling where it is required to install the sensors and that can be studied independently from the rest of the apartment. The identification of these independent zones can be done by an expert according to the necessity to localize people inside the dwelling area [1], [6]. The whole apartment can be decomposed in localization zones and in each zone independent sensor placement optimization problems can be defined and solved.

In Fig. 3, a simple flat composed by three zones is represented as example.

To formalize the optimization problem, it is necessary to discretize the dwelling area. To this aim, two independent grid scales are defined for each zone: 1) the grid scale $g_{c}$ discretizes the zones on the basis of the desired detection precision; 2) the grid scale $g_{p}$ defines the granularity of the sensors placement. In particular, the grid scales are defined in a 2-D space $(x, y)$ and it is possible to choose two different values of $g_{c}$ and $g_{p}$ for the two dimensions that are $\left(c_{x}, c_{y}\right)$ and $\left(p_{x}, p_{y}\right)$, respectively: $c_{x}$ and $p_{x}$ are the scales for the $x$ dimension, $c_{y}$ and $p_{y}$ are scales for the $y$ dimension, with $c_{x}, c_{y}, p_{x}, p_{y} \in \mathbb{N}^{+}$.

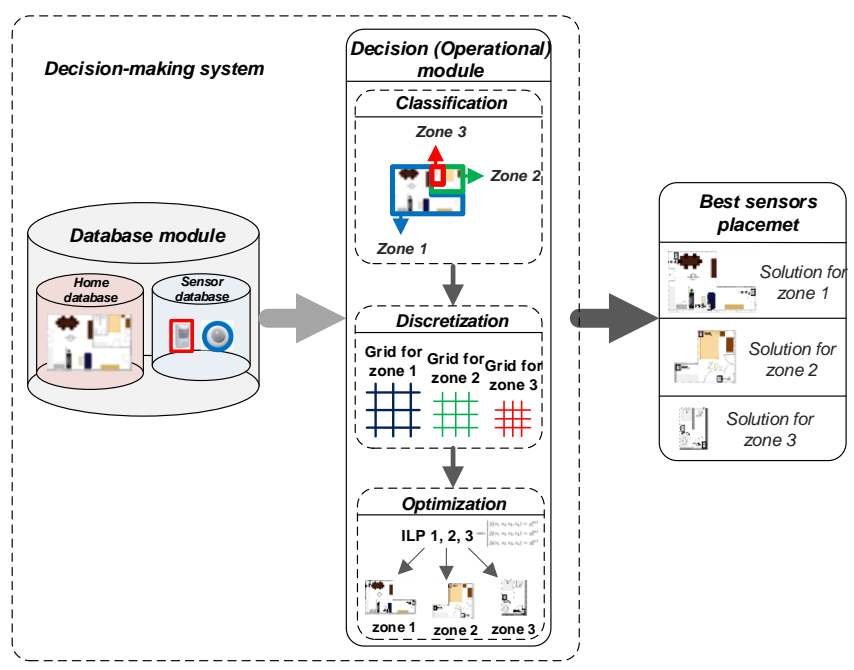

Fig. 3. The methodology for smart placement of sensors.

Now, to describe the flat discretization, the following sets are defined:

1) $\mathcal{N}=\left\{i \in \mathbb{N}^{+} \mid i=1, \ldots, N\right\}$ : set of points obtained by discretizing the considered zone within a 2-D grid scale $g_{c}$ that is suitable for obtaining the required coverage precision;

2) $O=\{i \in \mathcal{N} \mid i$ is a point of an obstacle $\}: O \subset \mathcal{N}$, set of points that are occupied by an obstacle;

3) $\mathcal{M}=\left\{l \in \mathbb{N}^{+} \mid l=1, \ldots, M\right\}$ : set of points obtained by discretizing the considered zone within a 2-D grid scale $g_{p}$ that is suitable for defining the precision of sensor placement;

4) $\mathcal{P}_{h}=\left\{j \in \mathcal{M} \mid j=1, \ldots, P_{h}\right\}: \mathcal{P}_{h} \subset \mathcal{M}$, set of points where sensors of type $h$ can be placed;

5) $\mathcal{P}=\bigcup_{h \in H} \mathcal{P}_{h}:$ set of points (with cardinality P) where sensors can be placed.

6) $\mathcal{K}=\{k \in \mathbb{N} \mid k=1, \ldots, K\}$ : set of angles corresponding to possible orientations of sensors.

It is remarked that the discretization of zones can be performed according to standard techniques [2].

\section{OPTIMIZATION PHASE SPECIFICATION}

In this section, the optimization phase is specified.

More in detail, the sensor placement optimization problem is defined by three ILP problems based on different objectives:

1. minimizing the cost of sensors.

2. minimizing the cost of sensors and maximizing the overlapping of detection areas. This goal allows finding the most robust solution among the ones at minimum cost in term of zone multi-covering [16].

3. minimizing the cost of sensors and the overlapping of detection areas. This is an important objective when performing location tracking within a house and it is necessary to distinguish which sensor has detected a movement, limiting detections overlapping [4]. 
The grid coverage is denoted by the following input binary variables, whose values are determined according to the Algorithm 1:

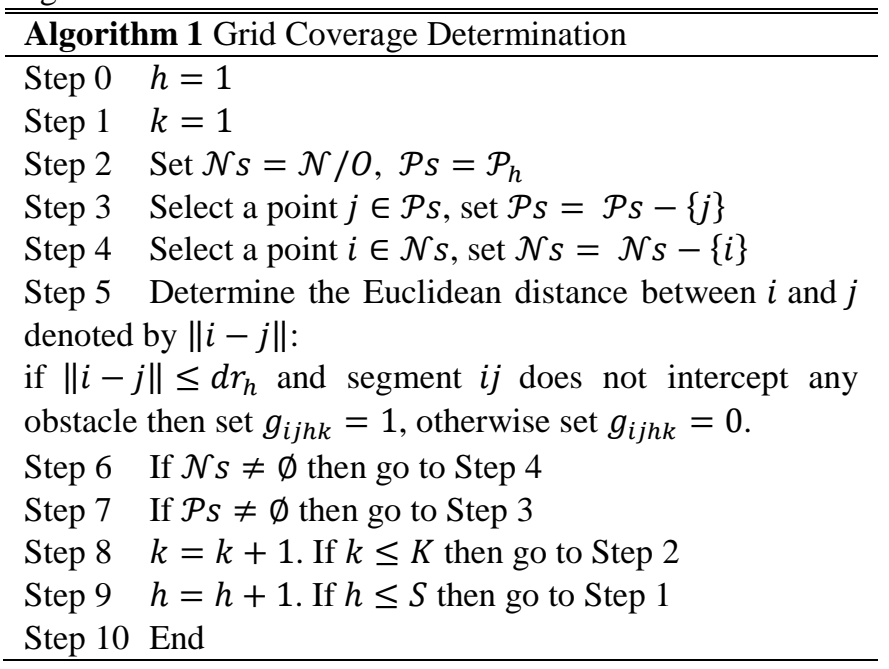

$$
g_{i j h k}=\left\{\begin{array}{c}
1, \text { if sensor of type } h \text { that is placed } \\
\text { at point } j \text { with orientation } k \\
\text { covers point } i \\
0, \text { otherwise } \quad \forall k \in \mathcal{K}, h \in H, j \in \mathcal{P}_{h}, i \in \mathcal{N} / O .
\end{array}\right.
$$

Note that $g_{i j h k}=0, \forall i \in O$ with $h \in H$ and $j \in \mathcal{P}_{h}$.

The decision variables are the binary variables

$$
x_{j h k}=\left\{\begin{array}{c}
1, \text { if sensor of type } h \text { is placed } \\
\text { at point } j \text { with orientation } k \\
0, \text { otherwise }
\end{array}\right.
$$

$\forall k \in \mathcal{K}, h \in H, j \in \mathcal{P}_{h}$.

Now, three ILP problems are formalized as follows to satisfy the three objectives.

\section{A. ILP problem 1}

The objective of ILP 1 is minimizing the total sensors number and cost, respecting specific coverage requirements:

$F_{1}=\min \sum_{j=1}^{P} \sum_{h=1}^{S} \sum_{k=1}^{K} c_{h} x_{j h k}$

subject to

$\sum_{j=1}^{P} \sum_{h=1}^{S} \sum_{k=1}^{K} g_{i j h k} x_{j h k} \geq a_{i}, \forall i \in \mathcal{N} / O$.

To express the coverage requirements of grid points $i \in$ $\mathcal{N} / O$ the following parameters are introduced: $a_{i} \in \mathbb{N}^{+}$if $i \in$ $\mathcal{N} / O$, where $a_{i}$ represents the minimum number of sensors that must cover the grid point $i ; a_{i}=0$ if $i \in O$, i.e., $i$ is an obstacle.

Equation (1a) minimizes the total sensors costs. Constraints (1b) are lower bounds for the minimum number of sensors $\left(a_{i}\right)$ that must cover the point $i \in \mathcal{N} / O$. The ILP problem (1a), (1b) is known in the related literature as the set multicover problem that is NP-hard [16].

\section{B. ILP problem 2}

The ILP 2 is a multi-objective problem with the aim of minimizing the number and cost of sensors and maximizing the overlapping of their detection areas. Thus, in order to formalize the ILP 2 , we consider $F_{1}(1 a)$ as the primary objective function and define a secondary objective function $F_{2}$, expressing the maximization of the overlapping of the detection areas:

$F_{2}=\max \sum_{i=1}^{N} \sum_{j=1}^{P} \sum_{h=1}^{S} \sum_{k=1}^{K} g_{i j h k} x_{j h k}$.

Then we optimize the two objective functions $F_{1}$ and $F_{2}$ in lexicographic order [17]: first function $F_{1}$ is minimized and then $F_{2}$ is maximized, while keeping $F_{1}$ at its minimum value.

The resulting formulation of ILP 2 is:

$\operatorname{lexmin}\left[F_{1}, F_{2}\right]$

subject to constraints

\section{ILP problem 3}

The ILP 3 is a multi-objective problem with the aim of minimizing the number and cost of sensors and minimizing the overlapping of their detection areas. Hence, in order to formalize the ILP 3 , we consider $F_{1}(1 a)$ as the primary objective function and define a secondary objective function $F_{3}$, expressing the minimization of the overlapping of detection areas:

$F_{3}=\min \sum_{i=1}^{N} \sum_{j=1}^{P} \sum_{h=1}^{S} \sum_{k=1}^{K} g_{i j h k} x_{j h k}$.

Then we optimize the two objective functions $F_{1}$ and $F_{3}$ in lexicographic order [17]: first function $F_{1}$ is minimized and then $F_{3}$ is also minimized, while keeping $F_{1}$ at its minimum value.

The obtained mathematical formulation of ILP 3 is:

$\operatorname{lexmin}\left[F_{1}, F_{3}\right]$

subject to constraints

\section{CASE Study}

In this section, a case study is presented to validate the proposed methodology on a specific dwelling. The map of the considered dwelling is shown in Fig. 4. Here we apply the proposed procedure to the highlighted localization zone that identifies the open space including the entrance hallway, the kitchen, the dining room and the living room.
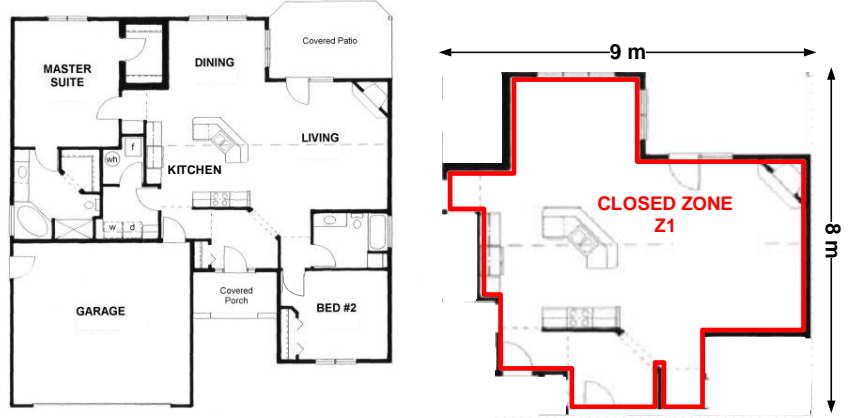

Fig. 4. The dwelling map and the considered localization zone. 
TABLE I

THE DATABASE OF MOTION SENSORS

\begin{tabular}{|c|c|c|c|c|c|c|c|c|}
\hline & \multicolumn{4}{|c|}{ Wall Sensors } & \multicolumn{4}{|c|}{ Ceiling Sensors } \\
\hline $\begin{array}{l}\text { Sensor } \\
\text { Features }\end{array}$ & $h=1$ & $h=2$ & $h=3$ & $h=4$ & $h=5$ & $h=6$ & $h=7$ & $h=8$ \\
\hline $\begin{array}{l}\text { Detection } \\
\text { radius }[\mathrm{m}]\end{array}$ & 6 & 9 & 6 & 9 & 6 & 9 & 6 & 9 \\
\hline $\begin{array}{l}\text { Detection } \\
\text { angle }\left[^{\circ}\right]\end{array}$ & 110 & 110 & 180 & 180 & 180 & 180 & 360 & 360 \\
\hline $\begin{array}{l}\text { Orientation } \\
(\text { start angle) } \\
\left.{ }^{\circ}\right]\end{array}$ & \multicolumn{4}{|c|}{$\begin{array}{c}0 / 60 / 120 / 180 / 240 / \\
300\end{array}$} & \multicolumn{2}{|c|}{$\begin{array}{c}0 / 60 / 120 \\
/ 180 / 240 \\
/ 300\end{array}$} & & \\
\hline Cost $[€]$ & 10 & 15 & 12 & 18 & 22 & 28 & 25 & 30 \\
\hline
\end{tabular}

The localization zone $Z_{l}$ is discretized by a grid $g_{c}=$ $\left(c_{x}, c_{y}\right)$ for zone coverage and a grid $g_{p}=\left(p_{x}, p_{y}\right)$, for sensor placement with $c_{x}=c_{y}=p_{x}=p_{y}=1 \mathrm{~m}$. In addition, the database of sensors is reported in Table I. Eight different types of sensors are considered, that are wall sensors $(h=1, \ldots, 4)$ and ceiling sensors $(h=5, \ldots, 8)$ with their detection capacities, possible orientations and costs. It is noted that the orientation is not relevant for $360^{\circ}$ ceiling sensors.

In the following the results of the application of ILP $1,2,3$ problems to the zone $Z_{l}$ are respectively provided. We set $a_{i}=$ $1 \forall i \in \mathcal{N} / O$, for all the three cases, which means that at least one sensor must cover each zone not representing obstacles. The ILP problems are solved by using a standard solver, i.e., GNU Linear Programming Kit [18].

\section{A. ILP 1 solution}

The ILP 1 problem is formalized and solved for the zone $Z_{l}$. The result gives a set $\mathcal{S}(a)$ of solutions that guarantees the respect of the coverage requirements at minimum cost [1].

$\mathcal{S}(a)=\left\{\begin{array}{l}\left(x_{1 w}, y_{1 w}\right)=(4,7) \text { and }\left(x_{1 c}, y_{1 c}\right)=(5,2) ; \\ \left(x_{2 w}, y_{2 w}\right)=(4,7) \text { and }\left(x_{2 c}, y_{2 c}\right)=(5,3) ; \\ \left(x_{31 w}, y_{31 w}\right)=(4,7) \text { and }\left(x_{32 w}, y_{32 w}\right)=(0,2) \text { and }\left(x_{33 w}, y_{33 w}\right)=(8,5) ; \\ \left(x_{41 w}, y_{41 w}\right)=(4,7) \text { and }\left(x_{42 w}, y_{42 w}\right)=(2,0) \text { and }\left(x_{43 w}, y_{43 w}\right)=(8,5) ; \\ \left(x_{51 w}, y_{51 w}\right)=(4,7) \text { and }\left(x_{52 w}, y_{52 w}\right)=(8,3) \text { and }\left(x_{53 w}, y_{53 w}\right)=(8,5) ; \\ \left(x_{61 w}, y_{61 w}\right)=(2,1) \text { and }\left(x_{62 w}, y_{62 w}\right)=(4,7) \text { and }\left(x_{63 w}, y_{63 w}\right)=(8,5) \text {. }\end{array}\right\}$

Each of the solution is composed by a combination of sensors. For brevity, Fig. 5 depicts only the last solution of the set $\mathcal{S}(a)$ that is given by the combined use of three sensors: a) a sensor of type 1 placed at coordinates $(2,1)$ with orientation $60^{\circ}$; b) a sensor of type 1 placed at coordinates $(4,7)$ with orientation $180^{\circ}$; c) a sensor of type 2 at coordinates $(8,5)$ with orientation $180^{\circ}$. Note that the grid coordinates are computed according to the methodology proposed in [1].

Finally, it is remarked that the total cost of each of the solution of set $\mathcal{S}(a)$ is $35 €$.

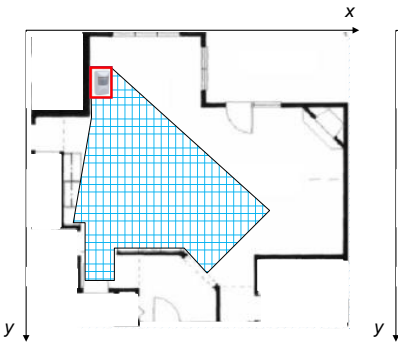

(a)

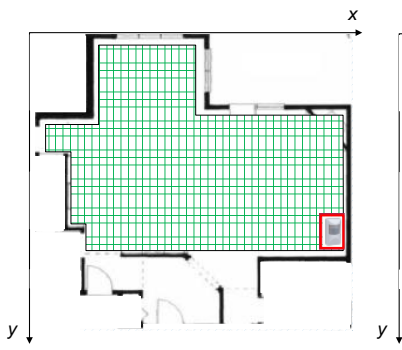

(c)

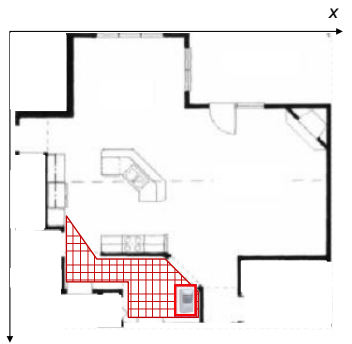

(b)

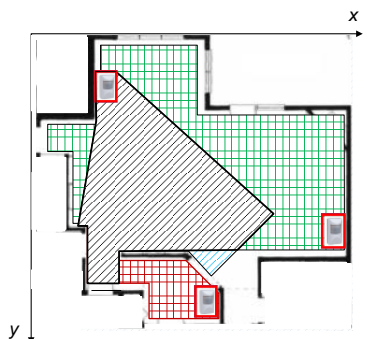

(d)
Fig. 5. The ILP 1 solution 6: (a) sensor of type 1 at $(2,1)$; (b) sensor of type 1 at $(4,7)$; (c) sensor of type 2 at $(8,5)$; (d) combination of the three sensors with overlapping of detection areas.

\section{B. ILP 2 solution}

The ILP 2 problem is formalized and solved for the zone $Z_{l}$. The obtained solution is the one of set $\mathcal{S}(a)$ that maximizes the overlapping of detection areas at minimum cost $(35 €)$. The solution is represented in Fig. 6 and it is given by the combination of three sensors: a) a sensor of type 1 placed at grid coordinates $(4,7)$ with orientation $180^{\circ}$; b) a sensor of type 1 placed at grid coordinates $(8,3)$ with orientation $180^{\circ}$; c) a sensor of type 2 placed at grid coordinates $(8,5)$ with orientation $180^{\circ}$.

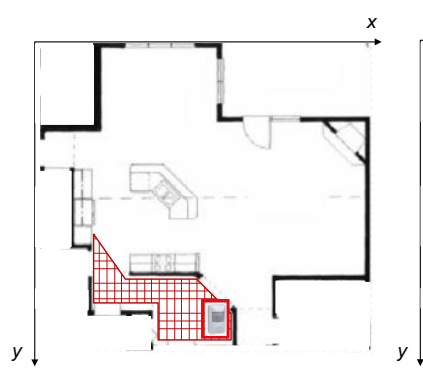

(a)

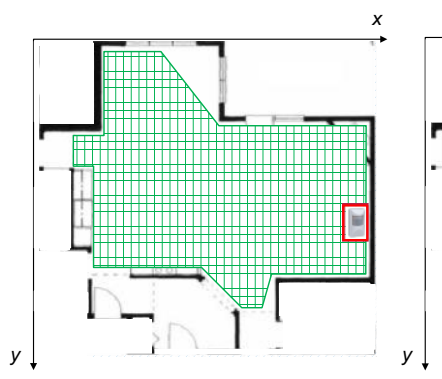

(c)

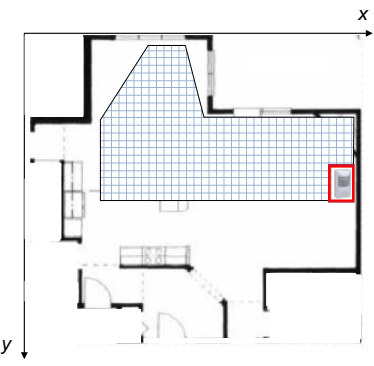

(b)

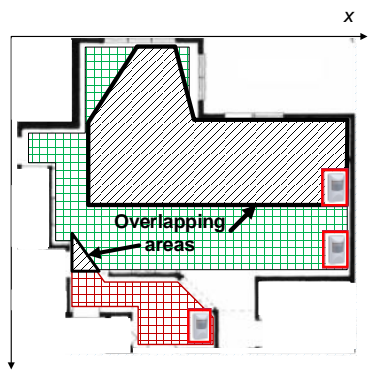

(d)
Fig. 6. The ILP 2 solution: (a) sensor of type 1 at $(4,7)$; (b) sensor of type 1 at $(8,3)$; (c) sensor of type 2 at $(8,5)$; (d) combination of the three sensors with overlapping of detection areas. 


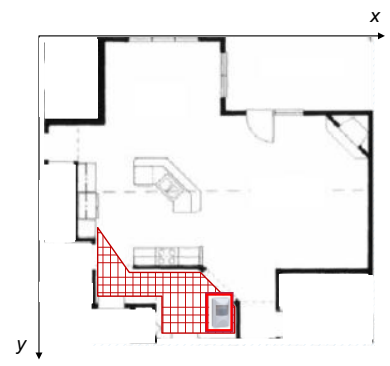

(a)

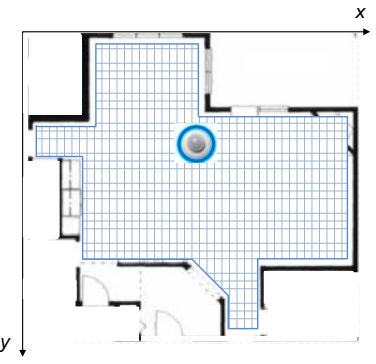

(b)

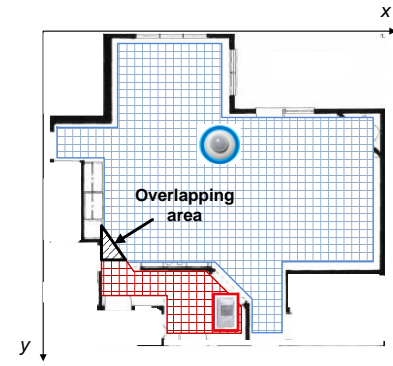

(c)

Fig. 7. The first solution of set $\mathcal{S}(a)$ : (a) sensor of type 1; (b) sensor of type 7; (c) overlapping area of the two sensors.

\section{ILP 3 solution}

The ILP 3 problem is formalized and solved for zone $Z_{l}$. The obtained solution is the one of set $\mathcal{S}(a)$ that minimizes the overlapping of detection areas at minimum cost $(35 €)$. The solution is given by the combination of two sensors, as it is shown in Fig. 7: a) a sensor of type 1 (Fig. 7a) placed at coordinates $(4,7)$ with orientation $180^{\circ}$ and $b$ ) a sensor of type 7 (Fig. 7b) placed at coordinates $(5,2)$ with orientation $300^{\circ}$.

In conclusion, the optimization phase of the proposed methodology can provide different solutions about sensors deployment, on the basis of the designer necessities. ILP 1 allows finding a set of optimal solutions that minimize the sensors cost, ILP 2 provides the solution that minimizes cost and maximizes the overlapping of detection areas and ILP 3 provides the solution that minimizes both the costs and the overlapping of detection areas.

\section{CONCLUSIONS}

This paper proposes a methodology for smart placement of motion sensors within a domestic environment. The methodology is implemented by a decision-making system and is composed by three phases: a classification phase to identify the zones to be analyzed, on the basis of the home geometry, a discretization phase to discretize the identified zones by using two dimensional grids: a grid for sensor placement and a grid for zone coverage. Moreover, an optimization phase allows satisfying different optimization objectives related to sensors cost and detections overlapping, for each localization zone of the house. A case study also is presented to validate the methodology on a specific zone of a home map. Future works will include other kinds of sensors, like pressure sensors, door barriers, etc., and will concern the sensors deployment optimization for daily activities recognition.

\section{REFERENCES}

[1] M. P. Fanti, G. Faraut, J.-J. Lesage and M. Roccotelli, "An Integrated Framework for Binary Sensor Placement and Inhabitants Location Tracking," in IEEE Transactions on System Man and Cybernetics: Systems, vol. PP, no. 99, pp. 1-7, September 2016.

[2] M. P. Fanti, M. Roccotelli, J. J. Lesage and G. Faraut, "Motion detector placement optimization in smart homes for inhabitant location tracking," in IEEE 21st International Conference on Emerging Technologies and Factory Automation (ETFA), Berlin, 2016, pp. 1-6.

[3] I. Vlasenko, I. Nikolaidis and E. Stroulia, "The Smart-Condo: Optimizing Sensor Placement for Indoor Localization," in IEEE Transactions on Systems, Man, and Cybernetics: Systems, vol.45, no.3, pp.436-453, March 2015.

[4] S. P. Emmons and F. Kamangar, "Evaluating the optimal placement of binary sensors," Int. J. Inf. Sci. Tech., vol. 3, no. 1, pp. 1-10, January 2013.

[5] A. N. Bishop, B. Fidan, B. D. O. Anderson, K. Do־gançay, and P. N. Pathirana, "Optimality analysis of sensor-target localization geometries," Automatica, vol. 46, no. 3, pp. 479-492, Mar. 2010.

[6] M. Danancher, J.-J. Lesage, L. Litz, and G. Faraut, "Online location tracking of a single inhabitant based on a state estimator," in Proceedings IEEE Int. Conf. on System Man Cybernetics (SMC), Manchester, U.K., Oct. 2013, pp. 391-396.

[7] A. L. Ballardini, L. Ferretti, S. Fontana, A. Furlan and D. G. Sorrenti, "An Indoor Localization System for Telehomecare Applications," in IEEE Transactions on Systems, Man, and Cybernetics: Systems, vol. 46, no. 10, pp. 1445-1455, Oct. 2016. doi: 10.1109/TSMC.2015.2503339

[8] A. M. Tabar, A. Keshavarz, and H. Aghajan, "Smart home care network using sensor fusion and distributed vision-based reasoning" in Proc. $4^{\text {th }}$ ACM Int. Workshop Video Surveillance Sensor Netw., Santa Barbara, CA, USA, 2006, pp. 145-154.

[9] S. A. Mehdi and K. Berns, "A survey of human location estimation in a home environment," in Proceedings of 23rd IEEE Int. Symp. Robot Human Interact. Commun., Edinburgh, U.K., Aug. 2014, pp. 135-140.

[10] H. Aghajan, J. C. Augusto, C. Wu, P. McCullagh, and J.-A. Walkden, "Distributed vision-based accident management for assisted living," in Pervasive Computing for Quality of Life Enhancement. Heidelberg, Germany: Springer-Verlag, 2007, pp. 196-205.

[11] J. Koch, J. Wettach, E. Bloch, and K. Berns, "Indoor localisation of humans, objects, and mobile robots with RFID infrastructure," in Proceedings of 7th Int. Conf. Hybrid Intell. Syst. (HIS), Kaiserslautern, Germany, Sep. 2007, pp. 271-276.

[12] M. Volkhardt, S. Mueller, C. Schroeter, and H.-M. Gross, "Playing hide and seek with a mobile companion robot," in Proceedings of 11th IEEERAS Int. Conf. Humanoid Robots (Humanoids), Bled, Slovenia, Oct. 2011, pp. 40-46.

[13] A. Gaddam, K. Kaur, S. C. Mukhopadhyay, and G. S. Gupta, "Selection and optimization of wireless sensors in a smart digital home for the elderly," in Proceedings IEEE Sensors, Christchurch, New Zealand, Oct. 2009, pp. 1382-1386.

[14] H. Ghayvat, S. C. Mukhopadhyay, X. Gui and J. Liu, "Enhancement of WSN Based Smart Home to a Smart Building for Assisted Living: Design Issues," 2015 Fifth International Conference on Communication Systems and Network Technologies, Gwalior, 2015, pp. 219-224.

[15] D. Basu, G. Moretti, G. Sen Gupta and S. Marsland, "Wireless sensor network based smart home: Sensor selection, deployment and monitoring," 2013 IEEE Sensors Applications Symposium Proceedings, Galveston, TX, 2013, pp. 49-54. doi: 10.1109/SAS.2013.6493555

[16] C. Chekuri, K. L. Clarkson, and S. Har-Peled, "On the set multicover problem in geometric settings," ACM Transactions Algorithms, vol. 9, no. 1, p. 9, 2012.

[17] Ehrgott, M, "Multicriteria optimization," Springer Science \& Business Media. 2006.

[18] GLPK (GNU Linear Programming Kit), Free Softw. Found. Inc., Boston,MA, USA, 2012. 